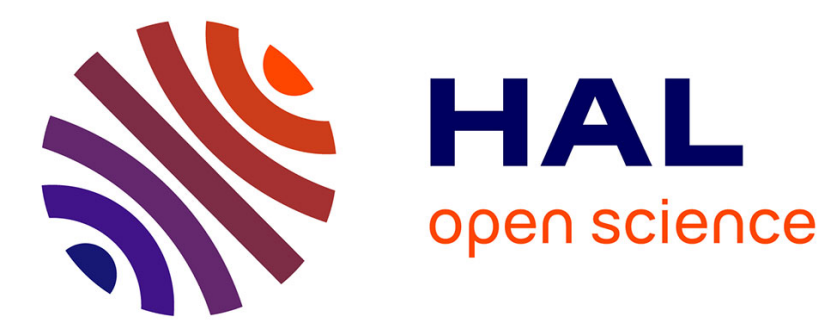

\title{
S'attacher à la politique. Carrières de jeunes socialistes professionnels \\ Lucie Bargel
}

\section{To cite this version:}

Lucie Bargel. S'attacher à la politique. Carrières de jeunes socialistes professionnels: Carrières de jeunes socialistes professionnels. Sociétés contemporaines, 2011, 84, pp.79-102. halshs-00724354

\section{HAL Id: halshs-00724354 \\ https://shs.hal.science/halshs-00724354}

Submitted on 20 Aug 2012

HAL is a multi-disciplinary open access archive for the deposit and dissemination of scientific research documents, whether they are published or not. The documents may come from teaching and research institutions in France or abroad, or from public or private research centers.
L'archive ouverte pluridisciplinaire HAL, est destinée au dépôt et à la diffusion de documents scientifiques de niveau recherche, publiés ou non, émanant des établissements d'enseignement et de recherche français ou étrangers, des laboratoires publics ou privés.

\section{(1) (1) $\$$}

Distributed under a Creative Commons Attribution - NonCommercial - NoDerivatives| 4.0 
Lucie Bargel

\section{S'attacher à la politique. Carrières de jeunes socialistes professionnels ${ }^{1}$}

Cet article propose de croiser la sociologie des effets biographiques de l'engagement et celle de la professionnalisation politique. Il s'agit en particulier de considérer l'occupation d'emplois politiques ${ }^{2}$ comme une possible «rétribution du militantisme », parmi d'autres, découverte dans le cours de l'action, et objet de valorisations variables, qui n'ont de sens qu'à travers les perspectives mouvantes des militants (Gaxie, 2005). L'approche interactionniste de la socialisation permet ainsi de tenir éloigné le finalisme qui caractérise au contraire les analyses fonctionnalistes, très répandu dans les commentaires et les analyses portant sur les jeunes partisans. Pour comprendre comment la professionnalisation politique devient objectivement possible et subjectivement désirable pour certains jeunes socialistes, il faut donc retracer le processus de socialisation qui la précède.

On propose ici de considérer leur engagement politique comme un processus d'unification des dispositions et des pratiques des membres du Mouvement des jeunes socialistes (MJS) (Becker, 1960; Bourdieu, 1979). L'analyse interactionniste de la socialisation professionnelle et déviante - permet d'explorer ce processus en mettant l'accent sur les pratiques objectives auxquelles apprennent à se livrer les militants enquêtés ainsi que sur les changements de perspectives subjectives que celles-ci engendrent et qui les rendent possible (notamment Becker et al, 1961). Olivier Fillieule utilise le terme « attachement » afin de « distinguer les logiques du maintien de l'engagement de celles qui président à l'enrôlement » (2005): on considérera ici leur professionnalisation en politique comme l'une des composantes du processus de maintien, voire d'intensification, de l'engagement de jeunes socialistes. A travers l'exploration de leur carrière au sein du MJS, il s'agit de comprendre comment certains deviennent «attachés » à la politique c'est-à-dire à la fois qu'ils l'apprécient positivement, qu'ils tiennent à y être loyaux l'attachement est un lien affectif et moral - et qu'il leur apparaît impossible de quitter ce sentier de dépendance - l'attachement est aussi une contrainte.

Cette perspective contribue également à élargir l'étude du personnel politique, en y intégrant les professionnels non-élus. L'attention académique à ces emplois a été faible jusqu’à présent - malgré quelques travaux récents (Courty, 2005 ; Aldrin, 2007 ; Le Lidec, 2008) -, et ceux-ci souvent pensés par défaut par rapport au cas « classique » du député (Best et Cotta, 2000),

\footnotetext{
${ }^{1}$ Je remercie Solenne Jouanneau, Catherine Leclercq, Julian Mischi, Julie Pagis et les lecteur-trice-s anonymes de la revue pour leurs contributions à l'amélioration de ce texte.

${ }^{2}$ On entend par "professionnels de la politique » l'ensemble de ceux qui « vivent pour et de la politique » (Weber, 1919)
} 
comme des «semi-professionnels» (Beyme, 1996) ou comme un «marché politique périphérique » (Offerlé, 2000). Pourtant, les lois de décentralisation puis sur la "démocratie de proximité », en renforçant les prérogatives des élus locaux ainsi que leurs moyens matériels, ont également contribué à la professionnalisation de leurs entourages, si bien qu'on peut estimer qu'aujourd'hui 7500 collaborateurs de cabinet et de groupes politiques sont rétribués sur les fonds des collectivités territoriales (Demazière et Le Lidec, 2008). L'actualité récente suggère aussi que leur importance politique est grandissante. Nos travaux sur l'application de la loi sur la parité montrent que les «fidèles » des têtes de liste exclus des postes électifs du fait de cette loi ont été replacés dans les cabinets et les services des exécutifs locaux (Achin, Bargel, Dulong, et al, 2007). Ces deux mouvements de (re-)qualification des collaborateurs d'élus portent vraisemblablement à la hausse le nombre de trajectoires professionnelles entièrement politiques, d'élus qui n'ont jamais eu d'autre emploi que politique, d'abord comme collaborateur, puis comme élu.

C'est pourquoi il est particulièrement intéressant d'étudier ces postes de professionnels de la politique non-élus du point de vue des trajectoires de ceux qui les occupent. Si ces emplois ne résument pas les positions professionnelles des «anciens » du Mouvement des jeunes socialistes ${ }^{3}$, ils constituent les principales possibilités de professionnalisation politique précoce (Gaxie, 2000). Faut-il les considérer comme des positions d'attente pour de jeunes hommes et femmes pas encore sélectionnés sur un marché des postes électifs de plus en plus clos (Lefebvre et Sawicki, 2006) ? Comme un lot de consolation pour ceux qui ont sacrifié leurs études au militantisme sans pouvoir prétendre occuper le «métier politique » pour lequel ils ont été formés? Passer par les carrières de ceux qui occupent ces postes de professionnels au service des élus, permet d'en restituer toute l'ambiguïté à la fois subjective (ce ne sont pas les positions dont rêvaient leurs titulaires, ils n'ont pas de "vocation») et objective (elles font l'objet d'une valorisation très variable tant au sein du monde politique qu'à l'extérieur).

Ces analyses reposent sur une enquête ethnographique menée auprès de membres du Mouvement des jeunes socialistes en 2003 et 2004 (Bargel, 2009). Pendant treize mois, j’ai participé à l'ensemble des activités (réunions, campagnes, manifestations, etc.) d'un groupe local du MJS, et mené des entretiens avec ses membres. J'ai également mené des observations et des entretiens au cours d'évènements nationaux (Congrès) et internationaux (camp d'été) de l'organisation. Les analyses présentées ici constituent également une première exploitation de

\footnotetext{
${ }^{3}$ A titre d'indication, presque 40\% des anciens membres du Bureau national du MJS entre 1975 et 1993 figuraient, fin 2007, dans l'annuaire Profession politique, ces 46 personnes se répartissant également entre professionnels de la politique élus et non-élus.
} 
l'enquête collective de sociologie du travail des élus locaux menée dans le cadre de l'ANR Prelat ${ }^{4}$ : celle-ci étudie le fonctionnement des exécutifs régionaux, à travers, notamment, des entretiens biographiques avec les vice-présidents, et leurs interlocuteurs politiques et administratifs (cabinet, groupes politiques, direction des services, etc.). Je tire en particulier profit du fait que certains enquêtés sont communs aux deux corpus.

Le choix d'extraire quelques «cas » de responsables nationaux du MJS, particulièrement contrastés du point de vue de leurs propriétés sociales, vise à éviter l'uniformisation que peut produire un raisonnement en termes de carrière, a fortiori quand il est présenté de manière succincte. Sur le terrain qui nous intéresse ici, il est spécialement important de saisir que l'appartenance à l'institution militante est tout à la fois productrice d'homogénéisation et de différenciation (Lagroye 2002 ; Bargel et Yon, à paraître). En effet, contrairement à la plupart des objets sur lesquels la notion de carrière est mobilisée, l'activité politique ne recouvre pas seulement un processus d'apprentissage, mais également des luttes concurrentielles, concurrence qui porte précisément sur la possibilité de poursuivre la carrière. Si la sélection produite par la socialisation au métier politique au sein du MJS n'est pas au cœur de cet article, passer par des cas individuels permet d'en montrer quelques modalités.

On s'attachera d'abord aux carrières au sein du Mouvement des jeunes socialistes des militants étudiés, afin de souligner que leur professionnalisation politique n'est qu'un aspect temporellement second - de la réorganisation de l'ensemble de leurs sphères d'activités sociales autour de la politique. On s'arrêtera ensuite en détails sur les conséquences professionnelles de leur engagement militant, et sur l'ambivalence de cette modalité spécifique de professionnalisation politique qu'est l'occupation de postes de professionnels non-élus.

\section{«Gérer son temps » : La centralité croissante de l'engagement}

Pour comprendre les effets de l'engagement dans une organisation de jeunesse de parti politique, il faut prendre en compte conjointement ses dimensions objectives et subjectives. Le processus d'apprentissage informel qui s'y déroule repose largement sur les relations de sociabilité entre pairs. Ces relations, vécues comme amicales mais non moins formatrices, soutiennent le franchissement de chacune des trois «phases » de la carrière de membre d'une organisation de jeunesse d'un parti ${ }^{5}$.

\footnotetext{
${ }^{4}$ Coordonnée par D. Demazière, l'équipe comprend L. Bargel, H. Buisson-Fenet, M. Catla, J. Dejond, S. Gardon, A. Healy, I. Lacroix, P. Le Lidec, G. Marrel, S. Nicourd et E. Verdier. Les entretiens mobilisés ici ont été conduits par l'auteure seule.

${ }^{5}$ Les deux premières phases de la carrière ne sont que résumées ici, elles sont analysées de façon plus détaillée in Bargel 2009.
} 
La première d'entre elles consiste à prendre goût aux activités proposées par l'organisation réunions de débat d'un côté, et actions de «terrain » (distributions de tracts, collages d'affiches, animation de meetings) de l'autre. Si la formation du goût pour ces pratiques est favorisée par des dispositions sociales et genrées incorporées dans des trajectoires (dispositions par exemple à parler en public, ou à aimer apprendre), elle repose également sur leur réalisation collective et l'intégration au groupe que celle-ci permet. Les membres les plus expérimentés de l'organisation tiennent ainsi un rôle essentiel dans ce processus : ils donnent à voir les modes légitimes de participation et encouragent leur adoption par mimétisme, facilitant de ce fait l'incorporation d'un «sens pratique ». Ils jouent également un rôle de sélection tacite - par l'incitation sélective de certains entrants - et active - par le repérage des bons profils, susceptibles de monter dans l'organisation. L'attention portée par les «initiateurs» aux entrants, est en effet modelée par la nécessité permanente de renouveler les «cadres », statuts d'autant plus transitoires qu'il s'agit d'ici d'une organisation de jeunesse ${ }^{6}$.

L'apprentissage continue, deuxièmement, par le ralliement à un sous-groupe, un courant plus ou moins formalisé, dans lequel les nouveaux se retrouvent. Ils sont ainsi socialisés, via l'attachement à des groupes restreints et électifs, à la concurrence interne à l'organisation et plus généralement aux affrontements et à la conflictualité politiques. C'est l'affirmation d'une loyauté, inséparablement stratégique, morale et affective, à leurs « initiateurs » qui préside à l'engagement dans la défense des positions et des prises de positions du sous-groupe. Et celle-ci fournit de nombreux savoir-faire adaptés aux formes spécifiques de la concurrence propre à l'organisation (faire valoir ses prises de positions dans les débats et les amendements de textes politiques, recruter à leur tour des alliés...). L'intégration progressive et collective dans ces relations de concurrence permet l'apprentissage d'une appréhension ludique de la conflictualité politique, qui en atténue la violence et offre même la possibilité d'éprouver un plaisir à jouer et à voir reconnaître sa maîtrise du jeu, son habileté. De plus, elle est créatrice de raisons d'agir spécifiquement adaptées et dessinées par l'organisation investie, et fournit alors un langage pour dire son ambition (par exemple « montrer que l'on peut faire mieux » que les concurrents).

La sociabilité entre pairs continue d'être essentielle durant la troisième phase de la carrière, qui consiste à s'attacher à la politique. La centralité croissante, parmi les activités sociales des individus, de l'engagement dans l'organisation de jeunesse se perçoit dans la manière dont celui-ci entre progressivement en concurrence avec deux autres registres d'activité auparavant centraux pour ces jeunes «hors-jeu socialement» (Bourdieu, 1978) ${ }^{7}$ : leurs relations affectives et

\footnotetext{
${ }^{6}$ On peut être membre du MJS à partir de 15 ans et jusqu'à 28 ans révolus.

$7 \mathrm{Du}$ fait du recrutement social élevé de l'organisation, ses membres font partie de ceux pour qui la jeunesse correspond à un desserrement des contraintes (poursuite d'études plus longue, recul temporel de la mise en couple
} 
leurs études. Cette «tension constitutive de l'engagement» (Gottraux, 2005) s'exprime le plus souvent, dans les entretiens et dans les conversations informelles observées, en termes de «gestion du temps", qui est une manière d'exprimer des difficultés croissantes à concilier plusieurs investissements dans différentes sphères d'activités

\section{Une sociabilité de plus en plus étendue et exclusive}

L'établissement de liens de sociabilité au sein de l'organisation participe et nourrit la prise de goût à ses activités puis l'intégration dans un de ses sous-groupes; elle est ainsi un facteur d'attachement, en douceur et de manière ludique, à l'activité politique. Cependant, quelle que soit l'intensité émotionnelle et affective de ces liens, lorsque ceux-ci en viennent à entrer en concurrence avec des relations amicales extérieures à l'organisation, ils sont évalués selon un principe de non-exclusivité. Il s'agit en somme de résister à un phénomène observable chez les membres les plus engagés dans l'organisation, l'engagement « total».

Alors que je demande aux cinq membres du bureau d'un groupe local de remplir en ma présence le questionnaire que je prévois de faire passer au Congrès de 2003, une question provoque leurs réactions : il s'agit d'indiquer la proportion de militants parmi ses amis. «Ça, c'est méchant!» «Ce n'est pas sympa pour les gens du BN [Bureau national]!» plaisantent-ils. "Oh oui, c'est fou, la dernière fois, je suis allé à l'anniversaire d'Amélie ${ }^{8}$ [ancienne responsable de ce groupe, alors Secrétaire nationale du MJS], il n'y avait que des gens du MJS !» Je comprends que ce qui n'est «pas sympa », c'est d'amener les membres du BN à indiquer que tous leurs amis sont militants, constat péjoratif pour ces responsables locaux qui, eux, se défendent de «n'avoir que ça dans la vie».

Ces mêmes réticences face au caractère exclusif de la sociabilité militante sont en réalité également partagées par ceux, plus engagés dans l'organisation, qui servent de contre-modèle. En effet, une seule personne a répondu à cette question que «tous» ses amis sont militants. Ce rapport relativement distancié à la sociabilité organisationnelle est en réalité largement ambigu : il exprime davantage une spécificité personnelle («moi, je ne m’y ferai pas prendre ») qu’une remise en cause des modalités d'engagement dans l'organisation. Cette ambiguïté est présente dans l'ensemble des propos des enquêtés à ce stade de leur carrière, qui disent refuser de fréquenter exclusivement des «camarades » tout en exprimant bien, par ce refus, qu'il s'agit d'une possibilité qu'ils ne perçoivent que parce qu'ils en sont très proches.

comme de la naissance d'enfants...), ce qui n'est évidemment pas sans rapport avec leur disponibilité pour l'engagement. A titre d'indication, 39\% des pères des membres du MJS interrogés en 2003 sont cadres et professions libérales, quand c'est le cas de 13,8\% des pères des 15-29 ans d'après une exploitation secondaire de l'enquête Emploi INSEE de 2002.

8 Tous les prénoms sont des pseudonymes. 
Ces réticences à l'intégration dans une sociabilité exclusive renvoient à deux logiques conjointes. Premièrement, les membres des organisations de jeunesse ne se sont pas entièrement libérés, au cours de leur engagement, des appréciations stigmatisantes sur l'activité politique, que font, par exemple, leurs amis non-militants («j'ai des amis qui croient que je fais partie d'une secte» explique ainsi une responsable fédérale) non sans ambivalence («ça intéresse tout le monde mais c'est tellement bizarre et chelou », «le côté papier tue-mouche de la politique», comme l'exprime un secrétaire national). Les relations affectives avec des personnes qui ne sont pas engagées en politique constituent ainsi un frein à l'intégration entière dans le groupe militant : pour continuer d'être «compréhensible» par ses amis, il vaut mieux avoir d'autres sujets de discussions que ses activités politiques. L'ambiguité des enquêtés, qui oscillent entre l'expression de leur volonté de ne pas «se couper du monde » et leur intégration croissante dans la sociabilité organisationnelle, marque ainsi la prégnance de l'étiquetage déviant de leur carrière ${ }^{9}$, qui les conduit à chercher à échapper à ce stigmate tout à la fois en se démarquant d'une sociabilité exclusive et en y trouvant refuge.

Les modalités de cette sociabilité concourent également, deuxièmement, à en faire une pratique déviante, dans la mesure où ces jeunes socialistes connaissent de ce point de vue une évolution inverse à celle des membres de leur classe d'âge. Les études statistiques montrent en effet que la stabilisation du statut matrimonial et professionnel réduit considérablement le volume des relations amicales et la fréquence des rencontres (Forse 1991). Les relations s'individualisent (on se rencontre séparément, à son domicile...) et la dissociation entre «amis» et « connaissances » conduit à la décontextualisation des premiers : les « amis » sont ceux rencontrés au temps de la «jeunesse » et conservés sous une forme davantage individualisée, tandis que les groupes (professionnels, notamment) dans lesquels s’insèrent les «adultes» ne produisent plus que des relation de «connaissance» et non des amitiés (Bidart, 1997 ; Bidart et Pellissier, 2002). Or, nos enquêtés connaissent précisément un phénomène inverse. Les relations qu’ils nouent dans l'organisation sont de plus en plus nombreuses au fur et à mesure qu'ils s'y engagent et en fréquentent différents échelons géographiques et hiérarchiques. Elles sont, de plus, des relations fondamentalement collectives et contextualisées, non seulement par l'organisation (les «camarades» socialistes) mais également par le «courant», qui est le lieu d'une sociabilité d'autant plus intense qu'elle tend à être exclusive à ses membres.

Ces expressions de distance individuelle révèlent donc aussi, en négatif, la force d'une sociabilité militante perceptible par tous et expérimentée au moins partiellement par la plupart

\footnotetext{
${ }^{9}$ Comparable en particulier à celle des musiciens de dance (Becker, 2002).
} 
des responsables du MJS. En effet, l'exposition publique de cette dernière est omniprésente dans les événements collectifs qui ponctuent la vie de l'organisation.

Chaque Congrès du MJS comprend une « cérémonie des adieux ». En 2003 comme en 2005, les responsables nationaux qui « font leur adieux » insistent sur l'intensité des moments qu'ils ont vécus dans l'organisation, et surtout sur les relations amicales et amoureuses qu'ils y ont nouées. En 2003, Julie entame son discours en remerciant le MJS pour lui avoir permis de «trouver l'homme de sa vie », présent lors de la cérémonie et membre des instances nationales en même temps qu'elle. De même, la présidente sortante remercie-t-elle en premier lieu son compagnon, lui-même engagé, « sans qui elle ne serait pas là aujourd'hui », comme son successeur, en 2005, rend hommage à son ex-compagne, Secrétaire nationale, la femme « la plus belle et la plus intelligente ». L’intensité des relations amicales entre les Secrétaires nationaux est elle aussi valorisée. Expression paradigmatique de la force de leur attachement personnel à l'organisation et à ses membres, de nombreux intervenants, hommes comme femmes, pleurent en faisant leur discours. Les larmes font explicitement partie intégrante du rituel. Johanne, en 2003, qui anime la cérémonie et voit partir « sa bande d'amies » insiste ainsi au micro sur le fait qu'elle "ne pleure pas ", comme si elle l'avait précédemment promis, alors même qu'elle est visiblement en larmes.

Le sens rituel de cette cérémonie est explicité par Amélie, à la tribune en 2005. Après avoir admis qu'elle «ne connaît pas la moitié d'entre vous » et supposé que « cela doit vous paraitre étrange de voir des gens que vous ne connaissez pas venir raconter leur vie », elle réaffirme néanmoins l’intérêt d'une telle exposition publique : «je crois que c'est malgré tout important que vous y assistiez, parce que c'est ça, aussi, le MJS et c'est tout cela que le MJS vous apportera ». Comme insiste à sa suite Johanne, qui fait à son tour ses adieux, «je ne peux même pas dire que le MJS a changé ma vie, j’avais 16 ans quand j'y suis entrée. Le MJS, c'est ma vie».

Par cette «cérémonie des adieux », il s'agit en quelque sorte de promettre aux membres plus jeunes de l'organisation qui y assistent des moments et des relations personnelles de plus en plus intenses au cours de leur engagement, et de valoriser la force de la cohésion des membres du MJS. Et en effet, font leurs adieux les anciens membres du Secrétariat national, c'est-à-dire les personnes les plus engagées dans l'organisation, qui incarnent ainsi la «bonne» forme de sociabilité militante.

\section{Une (re)qualification politique des études}

Comme les relations amicales extérieures à l'activité politique, l'investissement scolaire peut, lui aussi, être concurrencé par l'engagement militant, et ce de deux manières. La première est relativement comparable aux logiques qui président à rendre difficile la conciliation de sociabilités diverses: les investissements en temps et en énergie que demandent tant le militantisme que les études (et d'autant plus que ces jeunes engagés sont le plus souvent étudiants 
à des niveaux élevés de formation ${ }^{10}$ ) peuvent devenir concurrents. La nécessité parfois très concrète de «choisir » à quelle activité donner la priorité est ainsi à prendre en compte dans les trajectoires de professionnalisation des enquêtés. La seconde modalité de concurrence entre engagement et études est plus variable, en ceci qu'elle dépend du degré de proximité initiale des études poursuivies à la politique, susceptible tant de générer des conflits qu'au contraire de confirmer des affinités entre les études et l'activité politique (Le Bart et Merle, 1997).

Dans la mesure où ces jeunes sont dans leur grande majorité en cours d'études longues et en situation de réussite scolaire, ils accordent un prix important à l'obtention de diplômes élevés, et sont donc peu enclins à les sacrifier à l'activité politique. En fait, l'engagement au MJS produit une injonction contradictoire, qui leur demande de consacrer du temps et de l'énergie aux activités de l'organisation, d'être «militant», tout en valorisant particulièrement les détenteurs de compétences politiques spécialisées formalisées dans des titres scolaires. Les deux termes de cette injonction renvoient à deux filières concurrentes de professionnalisation politique, la filière militante et la filière centrale ${ }^{11}$, qui ne sont néanmoins pas exclusives en pratique. En réalité, l'engagement dans le MJS produit des encouragements vis-à-vis de ces deux modèles de compétence politique. En effet, la valorisation constante de compétences politiques cultivées dans l'organisation (au cours des réunions, lors des rédactions d'amendements ou de textes politiques, etc.) constitue une forte incitation à la réorientation des études, ou à la réinterprétation de celles-ci en termes politiques, donc à la poursuite d'une formation liée à l'activité politique professionnelle, en même temps que l'encouragement au dévouement à l'organisation conduit certains enquêtés à rechercher des cursus scolaires et universitaires considérés comme «faciles » (c'est-à-dire peu coûteux en temps).

Il est malaisé de distinguer les effets de l'engagement en la matière. En effet, un choix scolaire «politique » peut préexister à l'engagement dans l'organisation, mais il peut aussi être, au moment de l'enquête, le résultat de la reconstruction d'une cohérence biographique et politique. Emmanuelle, au moment où elle s'inscrit en droit, et alors qu'elle n'est pas encore entrée au MJS, développe ainsi une grille de lecture politique des différentes Universités parisiennes destinée à s’y orienter. La réputation politique (liée notamment à l'anticipation de discriminations raciales pour elle qui est noire) prend même le pas sur la réputation académique dans son refus de s’inscrire à

\footnotetext{
${ }_{10} 27 \%$ des membres du MJS sont titulaires au moins d'un diplôme de niveau Bac +5 , 35\% d'un diplôme de niveau $\mathrm{Bac}+3$, alors même que $60 \%$ d'entre eux sont en cours d'études.

${ }^{11}$ Permettant aux hauts-fonctionnaires des cursus inversés depuis les cabinets ministériels, ou même les ministères, vers un mandat électif, en vertu d'une compétence attestée par un diplôme, cette filière centrale, «mandarinale » ou « administrative » apparaît et prend une importance croissante sous la Vème République (François 1999, Gaxie 2000).
} 
Paris 2 Assas $^{12}$, et elle rejoint finalement Paris 10 Nanterre («plutôt bien, c'était plutôt ouvert, plutôt de gauche...»).

De mêmes phénomènes peuvent être observés concernant le choix des matières, tant avant l'entrée dans l'organisation que pour ceux qui ont commencé à s'y engager. Si le droit, l'histoire et la sociologie sont largement représentées parmi les disciplines investies par les jeunes militants, la science politique cumule proximité à l'activité politique et prestige social. Elle est en effet une filière de reconversion privilégiée des militants du MJS - et ce d'autant plus qu'elle s'enseigne à partir de la $3^{\text {ème }}$ année, accueillant ainsi des étudiants venus d'autres disciplines et notamment de l'histoire et du droit. La science politique est considérée comme une discipline « amie », d'abord parce que réputée « de gauche », mais aussi parce qu'elle constitue, aux yeux de ces jeunes engagés, une possibilité de cristalliser dans un diplôme leur «maîtrise pratique de la logique immanente du champ politique » (Bourdieu 1981). De plus, certains membres du MJS tentent de se réorienter vers cette voie d'accès au métier politique facilement identifiée que sont Sciences Po puis l'ENA. Au MJS Paris, ils sont ainsi nombreux à se présenter au concours d'admission en $4^{\text {ème }}$ année à Sciences Po. Bien qu'ils bénéficient des conseils et des propositions d'aide en matière de révision d'autres militants qui y sont ou y ont été étudiants (« je t’assure, ça n'est pas si dur, je t'aide si tu veux» encourage régulièrement Nathalie, elle-même en réorientation puisqu'elle a intégré Sciences Po après l'obtention d'un diplôme d'HEC), la plupart d'entre eux y échoue ${ }^{13}$.

Etant donné la forte sélectivité de Sciences Po, et plus encore de l'ENA, les divers $3^{\text {ème }}$ cycles professionnalisants plus directement encore orientés vers la pratique politique, qu'ils forment des collaborateurs d'élus locaux ou de parlementaires, ou des communicants, constituent alors une voie de reconversion plus largement accessibles à ces jeunes engagés. Les diplômes universitaires tels que le master 1 ou master 2 recherche de science politique sont mieux encore ajustés aux contraintes des militants, en ceci que, sanctionnant principalement une activité de recherche, ils leur permettent de maitriser leur emploi du temps. Certaines universités sont de plus identifiées, là encore grâce à l'expérience de leurs aînés, comme admettant relativement facilement que les étudiants militants prennent davantage qu'une année universitaire pour valider ces diplômes (et notamment Paris 1, qui cumule à cette qualité le «prestige » de la Sorbonne). Ainsi, certains endroits précis de l'université, d'autant mieux identifiés par les membres du MJS qu'ils fréquentent, au sein de l'organisation, des anciens étudiants de ces diplômes, leur offrent la possibilité de tenter de convertir leurs compétences pratiques en titre scolaire, et ainsi en

\footnotetext{
${ }^{12}$ Cette réputation est notamment liée à la longue présence du GUD, groupuscule d'extrême-droite, créé à Assas en 1968 par d'anciens militants d'Occident.

${ }^{13}$ En 2006, seuls 14 \% des 2623 candidats à l'entrée en master à Sciences Po ont été admis.
} 
ressources politiques qu'ils détiennent en propre (à la différence de leurs ressources «militantes » qui sont fondamentalement collectives), et en retour, de leur ouvrir de nouvelles voies d'activité politique professionnelle.

Ces réorientations scolaires sont inséparablement des réorientations professionnelles. Même s'ils ne (se) le formulent généralement pas ainsi, la cristallisation des compétences politiques des enquêtés dans un diplôme encourage la valorisation de ce titre scolaire dans la sphère d'activité politique professionnelle, sans en être une condition nécessaire.

\section{S'attacher à la politique professionnelle}

L'intensité des relations de sociabilité avec d'autres militants encourage également l'entrée dans la sphère d'activité politique professionnelle, d'une part parce qu'elle fournit des modèles d'individus déjà engagés dans la politique professionnelle, rendant ainsi ce métier pensable et envisageable, d'autre part parce qu'elle prodigue un réseau de relations, un capital social (Bourdieu, 1980), susceptible d'ouvrir des voies d'accès à ce type d'emploi. Le recrutement pour un poste de professionnel de la politique est en effet, pour ces jeunes militants, plus souvent le résultat d'une mobilisation collective de leur groupe de sociabilité - qui est inséparablement un groupe politique - que d'une initiative individuelle.

Ce que l'on rassemble sous le terme de "professionnalisation politique », l'occupation d'un emploi dont les activités relèvent de la politique, concerne en réalité deux phases qui diffèrent dans la manière d'envisager ce type d'emploi, l'une où s'il s'agit un emploi temporaire, l'autre où il s'agit d'une orientation professionnelle de long terme. Le premier cas concerne principalement des jeunes qui sont, en même temps qu'ils occupent ce type d'emploi, titulaires de responsabilités dans l'organisation de jeunesse: l'emploi politique peut alors être considéré comme un prolongement de l'activité militante qui lui préexiste, et comme un moyen accessoire de la financer. Cette perspective temporaire peut progressivement se transformer en orientation plus durable, mais cette évolution n'a rien de mécanique.

\section{Une perspective temporaire : prolonger et financer son engagement politique}

Leur parti fournit quatre postes de permanents au MJS, rémunérés au SMIC: la professionnalisation au sein de l'organisation au sens strict est ainsi très marginale. La tendance des permanents du MJS à habiter ensemble, en colocation, en banlieue parisienne, tient ainsi autant à l'intensité de leurs liens de sociabilité - qu'elle renforce en retour - qu'à des contraintes matérielles, en particulier pour la majorité d'entre eux qui n'est pas parisienne avant de rejoindre 
les instances nationales de l'organisation. Les autres membres de l'équipe nationale occupent ainsi ces responsabilités comme des activités bénévoles, à côté d'une autre activité professionnelle ou étudiante. Des membres du Bureau national du MJS peuvent également occuper des postes d'assistant parlementaire - mieux rémunérés ${ }^{14}$. Si le recrutement des membres du BN à ces postes peut là aussi être une préoccupation collective («là, c'est priorité aux membres du BN pour les postes d'assistants parlementaires en ce moment», m'indique ainsi Alain qui s'interroge sur son orientation professionnelle), il s'agit prioritairement de leur fournir une source de revenu, par un emploi de secrétariat et de gestion des relations avec les électeurs de la circonscription, emploi à temps partiel qui leur laisse le temps de se consacrer aux activités du MJS. Il s'agit plus rarement de mener des activités proprement politiques, notamment car les membres de la majorité du MJS appartiennent à un courant minoritaire du PS, Nouvelle gauche (NG), qui compte peu de parlementaires dans ses rangs.

En revanche, d'autres types de poste, liés aux réseaux politiques de Nouvelle gauche, sont, d'une part, plus directement accessibles aux cadres du MJS et, d'autre part, davantage susceptibles d'être investis en tant qu'emplois «politiques» et non seulement « alimentaires». Le réseau politique de NG s'étend en effet au-delà du MJS : ses membres sont présents dans de nombreux groupes syndicaux ou associatifs, dans l'ensemble dirigés vers les « jeunes », par le biais de ses cadres multi-positionnés. Ce réseau d'organisations liées à Nouvelle gauche est ainsi à la fois pourvoyeur d'emplois (et en particulier la Mutuelle des Etudiants, dont les postes - salariés de direction ne peuvent être occupés que par des militants étudiants élus (Jean 2005)) et pourvoyeur de sens politique dans l'occupation de ces emplois, faisant de ceux-ci des prolongements de l'engagement au MJS - et d'autant plus que les salariés de la Mutuelle reversent une partie de leurs indemnités au MJS ou à l'UNEF. Ces deux dimensions sont très sensibles dans le récit que fait Emmanuelle de son recrutement à la LMDE, alors qu'elle était en même temps membre du BN du MJS.

«La mutuelle, ce qu'il s'est passé, c'est que j’avais fini mon DEA de droit social, je cherchais du boulot, je ne trouvais pas, j’ai fait plusieurs mois de petits boulots (...). Le président de la mutuelle m'avait déjà vue dans d'autres débats, avait déjà vu que je m’intéressais aux questions de santé, ayant deux fois raté médecine, c'est quand même des questions qui m’intéressaient, et les questions de protection sociale, normal, en plus par ma formation, même si je ne suis pas spécialiste de la Sécu, droit social, droit du travail, aide sociale, c'est tous ces trucs-là. Donc il se dit, je pense, elle a plus ou moins des compétences un peu techniques sur ces questions-là, et en plus politiquement elle en pense un peu

\footnotetext{
${ }^{14}$ Le salaire médian des assistants parlementaires est de $1700 €$ (équivalent temps plein) par mois. Cf. le questionnaire recueilli auprès de 445 assistants parlementaires de la XII ème législature de l'Assemblée Nationale par le Collectif d'animation et de recherche sur le métier d'assistant (CARMA) : P. Aldrin, T. Barboni, L. Bargel, N. Canoves, B. François, J. Fretel, S. Guyon, I. Hajji, P. Le Lidec et J. Meimon.
} 
quelque chose. Donc il me demande. Je dois répondre en un week-end! A ce moment-là, j'étais en stage dans une organisation syndicale patronale, en plus! C'était pas rien, c'était la fédération de la bijouterie, joaillerie, orfèvrerie, et des pierres précieuses (rires). Mais côté patronal. J'avais encore un mois de stage à faire, stage pas supra-intéressant mais quand même formateur, et puis pas mal sur le CV. J'avais fait ça parce que j’avais déjà fait un stage chez un avocat assez militant, assez de gauche, j’avais fait un stage dans une entreprise publique, et là je me disais qu'il fallait aller voir ce qui se passe de l'autre côté, ça complète le CV. Donc j'en suis là, et puis je n'ai pas vraiment de perspectives après le stage. Je ne suis plus inscrite à la fac, donc je n'ai plus de convention de stage, je n’ai rien de spécial de prévu, et donc tombe ce truc. On m'explique un peu à quoi ça ressemble, au départ je ne sais pas vraiment quel est le rôle d'un administrateur. Et puis je me dis d'accord, et puis t'es indemnisé. Donc je réponds dans les trois jours. Je me renseigne un peu auprès des uns et des autres, qu'est-ce que c'est, quel engagement, moi d'accord je connais un peu mais je ne suis pas non plus experte, on ne me demande pas d'être experte, c'est aussi un truc militant, c'est mettre en œuvre aussi certaines choses qu'on dit, sur lesquelles on se bat, la transformation sociale, c'est aussi avoir un pied dans le mouvement social et faire des choses. C'est comme un levier la mutuelle, c'est ce que dit Nouvelle gauche en fait, de transformer la société dans le politique, dans l'associatif, dans le syndical, etc. Et là c'est une entreprise qui peut mettre concrètement, directement, en œuvre certaines de ces orientations. $\gg^{15}$

Un tel poste ne constitue ainsi pas nécessairement, aux yeux d'Emmanuelle, une orientation professionnelle vécue comme durable. Sa première vertu est de la sortir d'une situation de précarité professionnelle et matérielle. Mais cette "proposition» contrarie ses tentatives pour se constituer un $\mathrm{CV}$ qui ne soit pas exclusivement "militant», tentatives qui l'amènent à occuper une position professionnelle inconfortable, tant d'un point de vue politique (un syndicat patronal) que social (les pierres précieuses). Même si elle s'approprie la signification politique, proposée par son courant, d'un poste à la LMDE, elle n'abandonne que ponctuellement sa volonté de trouver un emploi hors de son réseau militant. Elle continue d'ailleurs à chercher un emploi «non-politique » pendant qu'elle est administratrice de la LMDE, sans rencontrer de succès - le jour de notre entretien, elle sort d'un entretien d'embauche où elle a été repoussée sous un prétexte qui lui semble «bidon», dans la mesure où les conditions annoncées au téléphone étaient différentes, ce qui tient vraisemblablement d'une discrimination raciale.

La fédération Léo Lagrange offre également aux membres du MJS des possibilités de reconversion de leur expérience militante, et ce pour plusieurs raisons. D’abord, elle emploie 7000 personnes par an, dont 3000 permanents, principalement pour des tâches d'animation

\footnotetext{
${ }^{15}$ Entretien avec Emmanuelle, 6 janvier 2006.
} 
auprès de jeunes, dans le cadre d'actions visant leur «intégration sociale », leur "éducation citoyenne ", etc., tâches pour lesquelles l'occupation de responsabilités au MJS peut être considérée comme une préparation pratique. De plus, la fédération propose un discours de lutte contre l'individualisme et les injustices, et de valorisation de «l'éducation populaire » qui présente de nombreuses affinités avec les positions politiques de ces militants de gauche. Enfin, un des délégués nationaux de cette fédération est membre de Nouvelle gauche. Autrement dit, différents aspects de leurs carrières au sein du MJS (apprentissage des dispositifs d'animation, formulation de discours politique, acquisition d'un capital social) se cumulent pour faire de Léo Lagrange un lieu privilégié de reconversion des membres du MJS.

\section{Se professionnaliser}

L'occupation de ces postes de "petits professionnels » peut ainsi n'être qu'une activité temporaire, liée par exemple à un mandat de direction de l'organisation de jeunesse, d'une durée de deux ans en moyenne. Une seconde phase, qui n'a rien d'automatique, consiste au contraire à faire de ces «jobs étudiants» politiques une orientation professionnelle durable. Celle-ci est plus ou moins progressive. Pour certains enquêtés, le passage à une activité politique professionnelle n’est précédé ni d'une (ré)orientation des études en ce sens, ni de «jobs » liés à leurs mandats. Ainsi, Damien était étudiant en école d'ingénieur en électronique durant son mandat d'Animateur fédéral du MJS. Son diplôme obtenu, et son mandat terminé, après un bref passage par le Secrétariat du Bureau national du MJS, il devient conseiller politique de Martine Aubry à la mairie de Lille. Les exemples de ce type sont nombreux. La fréquence de ces trajectoires de professionnalisation au sein de leur réseau de sociabilité contribue tout à la fois à rendre cette voie envisageable voire attrayante aux yeux des individus engagés et à leur en ouvrir concrètement la possibilité. Ce processus n'a cependant rien d'automatique et est fait de multiples ajustements et réévaluations. Il est d'ailleurs significatif de ce point de vue que ces postes de "petits professionnels» de la politique ne se disent pas sur le mode de la «vocation»: certes nos enquêtés «se sont toujours intéressés à la politique »; pour autant, lorsqu'il s'agit d'évoquer leur trajectoire professionnelle, hésitations, ambivalences, considérations matérielles sont bien présentes. Nous verrons d'ailleurs que même des enquêtés très engagés dans ce type de carrière professionnelle continuent d'exprimer des réticences, liées aux spécificités des postes occupés.

\section{Éprowver la force de son attachement}

Un élément déterminant dans ce processus de professionnalisation politique est le fait d'éprouver concrètement que les choix politiques et professionnels précédents ont, de fait, fermé ou du moins amoindri certaines opportunités d'orientation professionnelle, en même temps qu'ils 
en rendent d'autres pensables et réalisables. Autrement dit, et pour reprendre les termes de Becker (1960), il s'agit pour ces jeunes activistes de réaliser que leurs "paris adjacents » précédents les ont déjà partiellement engagés dans une carrière politique professionnelle.

Cette prise de conscience peut se faire lors de la confrontation avec des recruteurs, comme dans le cas d'Emmanuelle, qui peine à valoriser son activité à la Mutuelle des étudiants.

« [La mutuelle], c'est assez hybride. Tu vois, ce matin [en entretien d'embauche], on me dit "mais vous mettez ça comme une expérience professionnelle sur votre CV ?’ Mais c'est professionnel, je vous assure! C'est des trucs que je ne referais peut-être pas avant longtemps. En même temps, au niveau militant, t’as parfois l'impression qu'il y a des gens qui sont pépères à la mutuelle... il y a aussi cette culpabilité, un peu, sur, pas le confort, mais les indemnités, ailleurs tu n'es pas payé pour militer. $»^{16}$

Réciproquement, ses interactions avec d'autres militants lui rappellent qu'elle n'est plus tout à fait une «militante» (au sens de bénévole). Le caractère «hybride » de cette activité, qui n'est ni complètement reconnue comme une activité militante par les autres agents engagés, ni entièrement considérée comme une expérience professionnelle par les employeurs potentiels, marque bien la phase de transition dans laquelle se trouve Emmanuelle, qui continue de s'adresser à des recruteurs extérieurs au champ politique alors même que son $\mathrm{CV}$ est de plus en plus marqué par son engagement.

Les recruteurs non-politiques fonctionnent ainsi dans cet univers comme les «parents et épouses » des musiciens de danse chez Becker, en formulant des exigences qui ne reconnaissent pas d'autorité aux conventions sociales qui régissent le monde politique professionnel et rendent ainsi perceptible aux militants que «leur conception de ce qu'ils font n'est pas partagée par les autres membres de la société » (2002). Comme pour ces musiciens, la famille des militants joue également ce rôle, et ce d'autant plus qu'elle est socialement éloignée de l'univers politique, comme c'est le cas de la mère de Simon, aide-soignante. Cette dernière étiquette de manière particulièrement explicite les activités de son fils comme « déviantes » : non seulement son poste de président du MJS n’est «pas un vrai métier», mais surtout ses activités de cadre syndical et mutualiste étudiant sont susceptibles de le mener « en prison » : « elle ne comprend rien du tout », résume son fils.

Simon lui-même n'est pas tout à fait imperméable à ces appréciations stigmatisantes, renforcées par l'«affaire » de la MNEF, contemporaine de l'enquête puisque le procès se termine en juin 2006. Son rapport ambigu à son CV l'illustre : un document détaillant son "parcours universitaire », son « parcours militant » et son " parcours professionnel » est bien présent sur son

\footnotetext{
${ }^{16}$ Entretien avec Emmanuelle, 6 janvier 2006.
} 
ordinateur (et il me l'imprime), pourtant il explique ne "pas pouvoir le sortir» dans une démarche de recherche d'emploi - au contraire. Il s'agit donc d'un «CV militant » par opposition à un «vrai $\mathrm{CV}$ ». Un $\mathrm{CV}$ « non-militant» impliquerait en effet de transformer l'ensemble de son «parcours militant» en une mention de «responsabilités associatives étudiantes » parmi ses hobbies : il s'agit là d'une pratique courante des responsables du MJS. Ce «CV militant » comporte cependant déjà des tentatives de mise en conformité avec des attentes anticipées d'employeurs potentiels, et en particulier deux tentatives pour réduire le «trou» dans son parcours scolaire, qui reste néanmoins de 4 ans même avec ces «rafistolages». Ces arrangements indiquent bien que Simon considère que son parcours scolaire est illégitime, tout comme son parcours professionnel qu'il «arrange » également dans la mesure où le président du groupe «socialistes et apparentés » au Conseil de Paris dont il est le «conseiller» n’est autre que le député dont il a également été l'assistant parlementaire. En somme, Simon se sent contraint d'étoffer quelque peu artificiellement tous les aspects de son CV qui ne relèvent pas de son "parcours militant », et même ces tentatives de normalisation lui semblent insuffisantes pour en faire un «vrai CV»c'est-à-dire un CV qui permet de trouver un «vrai travail ». Par conséquent, il est contraint - et il le ressent comme une contrainte - de poursuivre son engagement dans l'univers politique, et dans une position inconfortable puisque, dans la mesure où il n'a guère de sortie possible en l'absence d'autres qualifications que «militantes », il doit se maintenir dans le jeu, c'est-à-dire à la fois continuer de jouer le jeu et accepter le compromis.

«C'est compliqué aujourd'hui, parce que je ne sais pas ce que je vais faire après [la fin de son mandat de président]. Parce que finalement ça fait presque dix ans que je fais ça, enfin que je suis dans le milieu, mais en tous cas depuis 1998 je ne fais vraiment que ça. Que ça, que ça, que ça, donc ça commence à faire longtemps... Et finalement plus tu passes de temps à l'intérieur, plus ton champ de perspectives se rétrécit aussi, parce que t'es obligé de repartir de zéro, à la fac, tout ça, c'est pas simple... $»^{17}$

Les contacts avec leurs parents et avec des employeurs non-politiques sont ainsi l'occasion pour ces jeunes en voie de professionnalisation politique d'éprouver leur décalage, décalage qui est double, puisque s'ils sont engagés dans des activités largement incompréhensibles aux yeux des personnes extérieures au champ politique, ils ne sont pas pour autant pleinement disposés à se considérer comme des professionnels de la politique. Emmanuelle est ainsi, selon ses propres termes, «au milieu» de deux normes professionnelles: elle continue en partie d'adhérer à des normes professionnelles qui veulent qu'elle soit recrutée pour des compétences «techniques», "scientifiques», objectives, et non sur «ce qu'elle est ou ce qu'elle pense par

${ }^{17}$ Entretien avec Simon, 20 novembre 2005. 
ailleurs », autrement dit pour son engagement politique, dont elle souhaite qu'il reste "parallèle » à sa vie professionnelle. Ses propos insistent en particulier sur son refus du « piston »,qu'elle doit sans cesse réaffirmer face à ses amis du lycée qui lui enjoignent au contraire d'en tirer profit, entretenant ainsi un stigmate fréquemment apposé sur le métier politique, alors même que par leurs propres trajectoires professionnelles ("ça fait cinq ans qu'ils bossent, ils achètent») ils la renvoient à la précarité de la sienne propre, qu'ils imputent d'ailleurs à ses activités politiques («tu perds ton temps »). L'ambivalence d'Emmanuelle est donc, au moins en partie, modelée par les appréciations portées par ses amis non-militants sur son engagement (« tu perds ton temps sauf si cela te permet d'être pistonnée pour trouver du travail »), elles-mêmes largement ambiguës d'une part, et d'autre part en opposition complète à la norme de désintéressement qui prévaut chez les membres du MJS.

De plus, en comparaison avec d'autres enquêtés, son refus de «dépendre» du militantisme tout comme ses préventions face à des «boulots qu'il faut pouvoir se permettre » ne peuvent se comprendre que rapportés à la trajectoire particulièrement accidentée de ses parents (migrants du Togo, ils perdent tous deux leur emploi au début des années 1980 alors qu'ils sont endettés, et n'en retrouvent qu'après plusieurs années de grande précarité notamment en termes de logement). Celle-ci préside, selon ses dires mêmes, à sa recherche d'une «sécurité » professionnelle et économique, parce que ses parents ne peuvent lui assurer une entraide matérielle dont bénéficient au contraire d'autres jeunes professionnalisés, mais surtout parce qu'elle cherche à consolider une ascension sociale («en termes d'estime de toi et de voir comment t'as avancé ») que semblait lui ouvrir son parcours d'excellence scolaire, dans lequel avait intensément investi sa famille. Or, sa professionnalisation politique se fait sur fond de réévaluation de ses ambitions d'ascension sociale et ses réticences sont ainsi inséparablement une crainte du rattrapage par ses origines - au double sens du terme puisqu'elle fait l'expérience de discriminations raciales à l'embauche. Alors que sa scolarité à Henri IV semblait pouvoir lui assurer une sécurité matérielle dont ont manqué ses parents, désormais la confrontation avec ses amis du lycée la renvoie à sa propre précarité professionnelle, malgré son niveau de diplôme élevé (DEA), qui la singularise autant que son engagement politique. Après un an d'emplois précaires et de stages, et un an à la Mutuelle, elle pressent partiellement son engagement déjà avancé dans la politique professionnelle (« si ça se trouve au final on va me proposer un autre truc pour lequel je vais encore dire "bon, allez, d'accord, j'y vais, mais que pour quelque temps" »).

Pour Simon comme pour Emmanuelle, leurs origines sociales pèsent sur leur trajectoire professionnelle et, en particulier, leur ferment des portes de sortie de la carrière politique dont bénéficient au contraire d'autres enquêtés - ne serait-ce que parce qu'ils ont les moyens de 
reprendre des études, par exemple. D'un autre point de vue, tous deux font preuve d'un sens pratique qui leur permet de saisir des opportunités d'emplois politiques qui les tiennent hors de la précarité, sans toutefois l'éloigner entièrement.

La vision de la professionnalisation politique présentée par le biais de ces deux exemples individuels peut sembler exagérément sombre : postes précaires, peu rémunérés, etc. D’une part, elle correspond aux postes auxquels peuvent prétendre ces enquêtés à ce moment de leur carrière dans les organisations de jeunesse. D'autre part, et c'est ce qui en fait l'intérêt, les entretiens ont eu lieu lors d'une période transitoire pour Emmanuelle comme pour Simon, au cours de laquelle ils commencent à envisager une professionnalisation politique à l'aune de ces postes, qui leur sont les plus directement perceptibles. Un an et demi après, en 2007, Emmanuelle devient juriste permanente d'une Union départementale de la CFDT. Elle a donc atteint sa "grande idée » initiale consistant à travailler dans un syndicat, et concilie engagement politique et stabilité professionnelle. Au même moment, Simon est attaché parlementaire d'un sénateur, et Délégué national du PS à l'insertion des jeunes, rattaché au Secrétariat national aux affaires sociales et à l'emploi dirigé par Martine Aubry. Il est également conseiller municipal de sa ville d'origine, et suppléant de la députée PS. Depuis le Congrès de Reims, il est directeur de cabinet de la Première secrétaire du parti. Autrement dit, leurs perspectives professionnelles se sont assez fortement élargies. S’ils n'envisageaient alors principalement que des postes politiques peu prestigieux, c'est certes en raison d'une certaine lucidité quant à leurs opportunités objectives à ce moment-là, mais également en vertu de la «scotomisation» des rétributions (Gaxie 2005) en vigueur au MJS, qui rend difficilement dicible, y compris à soi, le fait d'envisager des postes de professionnels de la politique plus élevés, et notamment des mandats d'élu.

\section{Des positions professionnelles incertaines}

L'ambigüité des enquêtés vis-à-vis de l'occupation de positions de professionnels de la politique non-élus ne tient pas seulement à la réévaluation de leurs ambitions scolaires et professionnelles ou à la faible valorisation de ces postes par leurs familles ou leurs employeurs non-politiques. Au sein même du monde politique, ces positions professionnelles font l'objet d'une valorisation instable, qui a des conséquences sur les conditions objectives de travail. Nous l'aborderons ici rapidement à partir du cas de deux collaborateurs d'élus, professions qui ajoutent à des conditions matérielles incertaines une dépendance politique forte à l'employeur. Il faut ajouter que, contrairement à Emmanuelle et Simon, Nathan et Ariane viennent de familles très privilégiées : cette origine sociale plus favorisée ne les rend guère moins sensibles à la précarité de leurs emplois et de leurs statuts. 
En effet, occuper une position professionnelle en vertu de son expérience et de son réseau politique signifie que celle-ci n'est pas nécessairement indexée sur le niveau de diplôme détenu, déconnexion qui peut permettre d'accéder à des positions plus élevées que dans le monde du travail non-politique, mais qui maintient aussi une incertitude forte sur la possibilité de se maintenir au même niveau hiérarchique. L'exemple des positions successivement occupées par Ariane au sein d'un Conseil régional est éloquent. Elle y entre pour remplacer la «plume» du président, alors qu'elle est responsable nationale du MJS, qu'elle n'a encore que le Bac et connait une situation d'échec universitaire. Après un an passé au sein du cabinet de la présidence de la Région, on lui propose d'être standardiste au local de campagne des régionales en 2004, ce qu'elle accepte. Devenue ensuite chargée de mission auprès du groupe PS du Conseil régional, et ayant repris en parallèle ses études jusqu'à obtenir un Master 2 recherche en science politique, elle se voit, à nouveau, proposer de s'occuper du courrier au local de campagne en 2010. A cette incertitude sur le niveau hiérarchique de la position occupée correspond une ambivalence très forte sur les compétences détenues.

«On est très polyvalent; moi je trouve que le monde politique, en tous cas le monde de professionnels de la politique, c'est encore un monde de grand décloisonnement, tu peux passer de conseiller aménagement à conseiller culture, tu peux passer de conseiller culture aux services, tu peux passer des services aux finances, parce que c'est un milieu dans lequel tes compétences techniques ne sont pas demandées. Majoritairement, on cherche des gens qui ont un certain sens politique qui peut, après, s'appliquer dans tous les secteurs. Mais c'est incompréhensible dans le privé, ça, de pas être sur un truc précis. Et je ne peux pas arriver en disant je peux faire du logement, je peux faire autre chose, ce qui compte c'est mon sens politique, ils en ont rien à foutre. Or aujourd'hui c'est ma seule, c'est ma vraie compétence, parce que je pense que je bosse bien, mais c'est la seule. (...) J'ai refait mon CV là complètement et j’ai du mal à le finir, et j'ai essayé de le faire justement sur le thème des compétences, et donc j’ai mis analyse, conseil, formation. C’est un pipeau complet! Je suis pas conseillère, je suis pas formatrice, je suis pas analyste. Et en même temps c'est ce que je fais toute la journée. Donc on a le sentiment, tous, d'être des vrais imposteurs. $\gg^{18}$

Si Ariane refait son CV et se pose avec autant d'acuité la question de ses compétences professionnelles, c'est aussi parce que, huit ans après être entrée à la Région, elle envisage de quitter cet univers professionnel. Et pourtant les conditions matérielles de travail dont elle bénéficie au moment de l'entretien sont vraisemblablement supérieures à celles qu'elle pourrait obtenir ailleurs ; elle en est si consciente qu'elle refuse une augmentation, « parce qu'on s'habitue

${ }^{18}$ Entretien avec Ariane, 8 décembre 2009. 
très vite au confort, que je sais qu'il faut que je parte, et que je sais que je partirai plus facilement à 3000 [euros par mois] qu’à 4 ».

On pourrait imaginer que Nathan, lui aussi ancien responsable national du MJS, et devenu en 2010 directeur de cabinet du président de la même Région après avoir été son conseiller politique dans le précédent cabinet, bénéficie d'un rapport plus enchanté à sa trajectoire politique et professionnelle, dans la mesure où, outre son poste actuel, il cumule des ressources sociales personnelles (contrairement aux précédents, c'est un jeune homme blanc issu d'un milieu très favorisé) et des capitaux politiques importants (il est aussi «numéro 2 » de sa fédération départementale du Parti socialiste et élu municipal). Mais ses différentes positions ne sont pas parfaitement compatibles : comment en effet être un leader politique, et en particulier, dans son cas, participer à la direction d'un courant du Parti socialiste, quand on est en même temps au service d'un autre leader politique, le président d’une Région ? D’un côté, « ma légitimité n’est pas une légitimité administrative, mais une légitimité politique c'est-à-dire que c'est au nom de ce que j’ai pu faire dans le parti autour de Pierre [prénom du président] qu'on m'a choisi » ${ }^{19}$. Mais de l'autre côté, le fait même de le recruter en tant que directeur de cabinet entache ses capacités à entretenir cette forme de légitimité politique : «Si demain je prends la parole dans les instances du parti, beaucoup de gens vont considérer que je parle pour Pierre, et donc je me vois pas tenir les deux bouts. Mon problème c'est qu'aujourd'hui je suis passé plus du côté du collaborateur, c'est vrai que ma militance personnelle est beaucoup plus contrainte.» Si Nathan exprime assez clairement cette contradiction et ce que ce poste lui «coûte», c'est finalement la progression professionnelle qui l'emporte.

«Il faut le reconnaittre, par rapport à ma carrière professionnelle, c'est aussi un changement de... tu rigoleras en écoutant cette cassette si je suis à Pôle emploi, mais en réalité, ça me change de statut. Voilà. Je passe du Pôle emploi aux chasseurs de tête, en réalité. Ce qui est quand même pas quelque chose de négligeable, y compris dans la perspective de 2012 aussi. Mais ça veut dire que demain, peut-être, je serai plus légitime sur mon boulot professionnel que sur mon boulot politique. C'est un peu bizarre. Mais c'est vrai que j'ai un DEA [de science politique], j’ai jamais commencé ma thèse, j’ai toujours été sur des emplois précaires et j’ai trouvé ça très bien comme ça, j’ai jamais douté de ma capacité à rebondir, mais c'est vrai que là je passe un cap. Et je suis à l'âge [32 ans] où c'est plutôt utile d'emmagasiner ce genre d'expérience. $»^{20}$

Pour Nathan comme pour les autres, devoir son poste à un capital militant et non directement à un diplôme créé précarité et incertitude professionnelles, jusqu'au paradoxe de devoir entamer ses capacités militantes afin de stabiliser sa position professionnelle.

\footnotetext{
${ }^{19}$ Entretien avec Nathan, 15 juillet 2010.

${ }^{20}$ Idem.
} 
Les modalités d'occupation de ces postes de professionnels de la politique sont ainsi façonnées à la fois par les carrières dans lesquelles ils s'inscrivent et par leur institutionnalisation inachevée (incertitude sur la qualification requise, sur leur niveau dans la hiérarchie organisationnelle, etc.) Alors que les approches en termes « d'entrées en politique » (1996) la font souvent coïncider avec l'accès à un premier mandat électif, on mesure l'intérêt de passer par l'amont, par la socialisation à l'engagement politique, pour comprendre à la fois les positions politiques (professionnelles et/ou électives) occupées mais aussi les manières de les occuper et les façons de fabriquer des trajectoires politiques et professionnelles. Ceci sans négliger ce que ces socialisations doivent à l'état du champ politique dans lequel elles ont lieu - ici, à la clôture du marché des postes électifs, a fortiori nationaux, pour de jeunes socialistes.

On aurait aussi pu souligner l'intérêt de passer par l'amont des carrières politiques en insistant sur ce qu'elles permettent de saisir des lignes de démarcation qui séparent ceux qui ont été socialisés à l'engagement politique dans une organisation de jeunesse socialiste (MJS, mais aussi UNEF) et les autres. C. Bachelot (2008) montre ainsi que les premiers tendent à occuper des rôles similaires (de stratège et de négociateurs de courant) au sein de la direction du PS depuis une dizaine d'années. Au sein des conseils régionaux étudiés, on a également pu voir à quel point les similarités de socialisation au métier politique rassemblent anciens du MJS - alors même que certains sont élus et d'autres pas, et alors même qu'ils peuvent être membres de courants concurrents - et les opposent à ceux qui doivent plus directement leurs positions à une compétence «technique », certifiée par un diplôme spécialisé ou une expérience professionnelle. Leur socialisation politique si ce n'est commune, du moins similaire, les porte en effet à défendre la valeur des ressources militantes - et en particulier à en regretter la trop faible valorisation partisane à leur yeux (Lefebvre 2011). Leurs conceptions partagées du «métier», de ce qui est «politique» ou non, structurent ainsi les équipes de travail au moins autant que les règles formelles d'organisation du travail au sein des institutions politiques. 
ACHIN C., BARGEL L., DULONG D., et al., 2007 Sexes, genre et politique, Paris : Economica.

ALDRIN P., 2007 «Si près, si loin du politique. L'univers professionnel des permanents socialistes à l'épreuve de la managérialisation », Politix, nº79, p. 177-203.

BACHELOT C., 2008 «Groupons-nous et demain... »: Sociologie des dirigeants du Parti socialiste depuis 1993. Thèse de science politique. IEP de Paris.

BARGEL L., 2009 Jeunes UMP / Jeunes socialistes. Lieux et processus de socialisation politique. Paris : Dalloz.

BARGEL L., YON K., à paraittre Configurations politico-syndicales et production de normes militantes : les cadres de l'UNEF et du MJS depuis les années 1970, in : Defaud N., Llobet A. Dir., La condition politique des syndicats. Approche comparée. Paris : L'Harmattan.

BECKER H., et al., 2007 [1961] Boys in white : Student Culture in Medical School, New Brunswick : Transaction Publishers.

BECKER H., 1960 Notes on the concept of commitment. The American Journal of Sociology, vol. $66, \mathrm{n}^{\circ} 1$, p. $32-40$.

BECKER H., 2002 [1963] Outsiders. Etudes de sociologie de la déviance, Paris : Métailié.

BEST H. et COTTA M., 2000 Parliamentary Representatives in Europe 1848-2000 Legislative Recruitment and Careers in Eleven European Countries, Oxford: Oxford University Press.

BEYME K., 1996 The concept of political class: a new dimension of research on elites ? West European Politics, vol. 19, n 1, p. 68-87.

BIDART C., 1997 L'amitié, un lien social, Paris : La Découverte.

BIDART C. et PELLISIER A., 2002 Copains d'école, copains de travail. Evolution des modes de sociabilité d'une cohorte de jeunes, Réseaux, nº 115, p. 17-49.

BOURDIEU P., 1978 La “jeunesse” n’est qu’un mot. Entretien avec Anne-Marie Métailié, In Les jeunes et le premier emploi, Paris : Association des Ages, p. 520-530.

BOURDIEU P., 1979 La distinction : critique sociale du jugement, Paris : Minuit.

BOURDIEU P., 1980 Le capital social: notes provisoires, ARSS, vol. 31, nº 1, p. 2-3.

BOURDIEU P., 1981 La représentation politique. Eléments pour une théorie du champ politique, ARSS, n 36/37, p. 3-24.

COURTY G. (dir.), 2005 Le travail de collaboration avec les élus, Paris : M. Houdiard.

DEMAZIERE D. et LE LIDEC P., 2008 La politique, un objet pour la sociologie du travail. Sociologie du travail, vol. 50, n², p. 137-146.

« Entrées en politique », Politix, 35, 1996.

FILLIEULE O., 2005 Temps biographique, temps social et variabilité des rétributions. In Fillieule O. (dir.). Le désengagement militant. Paris: Belin. p. 17-48. 
FORSE M. 1991 Les réseaux de sociabilité : un état des lieux, L'année sociologique, n 41, p. 247 262.

FRANCOIS B., 1999 Le régime politique de la Vè République, Paris: La Découverte.

GAXIE D., 2000 La démocratie représentative. Paris : Montchrestien.

GAXIE D, 2005 Rétributions du militantisme et paradoxes de l'action collective, Revue Suisse de Science Politique, vol. 11, $\mathrm{n}^{\circ}$ 1, p. 157-188.

GOTTRAUX P., 2005 Autodissolution d'un collectif politique. Autour de Socialisme et Barbarie. In Fillieule O. (dir). Le désengagement militant. Paris : Belin, p. 75-94.

JEAN A., 2005 Des étudiants atypiques : les élus du bureau national de la mutuelle des étudiants. La LMDE "une mutuelle gérée par et pour des étudiants », mémoire de maittrise de science politique, Université Paris 1.

LAGROYE J., 2002 L'institution en pratiques. Revue Suisse de Science Politique, vol. 8, n 3-4, p. 114-128.

LE BART C. et MERLE P., 1997 La citoyenneté étudiante. Intégration, participation, mobilisation. Paris : PUF.

LE LIDEC P., 2008 Les députés, leurs assistants et les usages du crédit collaborateurs. Une sociologie du travail politique. Sociologie du travail. vol. 50, n² 2. p. 147-168.

LEFEBVRE R. et SAWICKI F., 2006 La société des socialistes. Le PS aujourd'bui, Broissieux: Ed. du Croquant.

LEFEBVRE R., 2011 Les primaires socialistes. La fin du parti militant, Paris : Raisons d'agir.

OFFERLE M., 2000 [1987] Les partis politiques, Paris : PUF.

WEBER M., 1963 [1919] Le savant et le politique, Paris : Union Générale d'Editions. 